\title{
Exposição a trauma e transtorno de estresse pós-traumático em usuárias de crack
}

\author{
Trauma exposure and posttraumatic stress disorder in crack cocaine users
}

Saulo Gantes Tractenberg', Thiago Wendt Viola', Caroline Silva de Oliveira Rosa', Julia Medeiros Donati', Ingrid D'Avila Francke², Julio Carlos Pezzi ${ }^{3}$, Rodrigo Grassi-Oliveira ${ }^{1,4}$

\section{RESUMO}

Objetivo: Verificar a ocorrência de trauma e transtorno de estresse pós-traumático (TEPT) em uma amostra de mulheres dependentes de cocaína tipo crack. Método: A amostra foi composta por 99 mulheres, entre 18 e 52 anos, internadas em uma unidade de desintoxicação e extensamente avaliadas por meio da SCID-I e a ASI-6. Resultados: Verificou-se uma taxa de exposição a trauma de $86,9 \%$ entre mulheres dependentes de cocaína tipo crack. A taxa de TEPT na amostra foi de $15,1 \%$. Os clusters de revivescência e hiperexcitabilidade foram os mais frequentes - 24,4\% e 20,9\% respectivamente. Entre os tipos de eventos relatados, os mais frequentes foram sofrer agressão/abuso físico e ser testemunha de violência a outros. Conclusão:

\section{Palavras-chave}

Trauma na infância, cocaína, abuso de substância, comorbidade.

\section{Keywords}

Childhood trauma, cocaine, substance abuse, comorbidity.
Os resultados sugerem uma frequente exposição a eventos traumáticos. Com relação à idade da experiência traumática, sugere-se que as usuárias expostas a trauma durante a infância e adolescência apresentam um início do uso de drogas em idades mais precoces que aquelas cujo trauma ocorreu na vida adulta.

\section{ABSTRACT}

Objective: To determine the occurrence of trauma and posttraumatic stress disorder (PTSD) in a sample of women addicted to crack cocaine type. Method: The sample comprised 99 women, between 18 and 52 years admitted to a detoxification unit and extensively assessed by SCID-I and ASI-6. Results: There was a trauma exposure rate of $86.9 \%$ among women addicted to crack cocaine type. The rate of PTSD in the sample was $15.1 \%$. The clusters of reexperiencing and hyperarousal were the most frequent, $24.4 \%$ and $20.9 \%$ respectively. Among the types of events reported most frequently were suffering assault/physical abuse and witnessing violence to others. Conclusion: The results suggest a frequent exposure to traumatic events. With regard to age of the traumatic experience, it is suggested that users exposed to trauma during childhood and adolescence showed a beginning drug use at earlier ages than those whose trauma occurred in adulthood.
1 Pontifícia Universidade Católica do Rio Grande do Sul (PUCRS), Núcleo de Estudos e Pesquisa em Trauma e Estresse (NEPTE). 2 Sistema de Saúde Mãe de Deus.

3 Universidade Federal de Ciências da Saúde de Porto Alegre (UFCSPA), Programa de Pós-Graduação em Ciências da Saúde.

4 Instituto de Pesquisas Biomédicas (IPB).

Endereço para correspondência: Rodrigo Grassi Oliveira

Pontifícia Universidade Católica do Rio Grande do Sul

Programa de Pós-Graduação em Psicologia e Instituto de Pesquisas Biomédicas

Av. Ipiranga, 6681, prédio 11, sala 936

90619-900 - Porto Alegre, RS, Brasil

E-mail: rodrigo.grassi@pucrs.br 


\section{INTRODUÇÃO}

A dependência química à cocaína tipo crack tem preocupado diversos setores da saúde pública, mostrando-se como um sério problema na realidade brasileira. Configura-se uma epidemia, se considerarmos que a dependência dessa droga vem crescendo exponencialmente na última década'. Nesse sentido, estudos que visem desenvolver conhecimento sobre a dependência de substâncias psicoativas, principalmente no que diz respeito aos fatores de vulnerabilidade, poderiam contribuir para ações preventivas no que tange aos prejuízos e sintomas associados a esse quadro. Particularmente, o usuário de cocaína tipo crack expõe-se a diversas situações de risco, que são potencialmente estressoras e traumáticas, como condições de violência e acidentes².

O modelo de vulnerabilidade da dependência química induzida pelo estresse constitui um dos mais extensamente estudados, pois agrega evidências que vão de pesquisas ani-

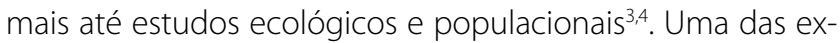
pressões máximas desse modelo seria a ocorrência do transtorno de estresse pós-traumático (TEPT), que torna ainda mais complexas as possibilidades de intervenções em indivíduos com transtornos relacionados ao uso de substâncias (TRUS) ${ }^{4,5}$.

Estima-se que a coocorrência de TRUS e TEPT seria de 20\% a 55\% ${ }^{6-11}$, embora alguns estudos demonstrem que essa taxa pode variar amplamente, situando-se de $11 \%$ até $91 \%^{12-14}$, o que é consideravelmente superior às taxas observadas na população em geral (1\%-9\%). Além disso, dados de prevalência indicam que $75 \%-99 \%$ dos pacientes adictos relatam terem sidos expostos a algum evento estressor durante o seu desenvolvimento $2,11,14,15$. Esses estudos também apontam que, em consequência dessa exposição, 59\% dessa população apresentam sintomas significativos de TEPT, principalmente entre populações femininas ${ }^{2,4,14}$. A hipótese para a ocorrência mais frequente de sintomas pós-traumáticos em mulheres possivelmente deve-se a fatores como maior suscetibilidade dessa população a desenvolver sintomas de TEPT e maior vulnerabilidade de exposição a determinados tipos de eventos como abuso sexual, violência física, sendo essas consideradas as principais experiências traumáticas que predispõem ao TEPT16-18.

No que se refere aos sintomas pós-traumáticos, constatou-se que pacientes diagnosticados com TEPT e dependência de substâncias em comparação com aqueles apenas acometidos pelo TEPT possuem uma ocorrência maior de sintomas relacionados à excitabilidade (cluster D) ${ }^{19}$. Esses estudos sugerem que os sintomas de excitabilidade decorrem do aumento dos níveis de ansiedade em períodos tanto de uso ativo quanto de abstinência de substâncias ${ }^{20-23}$. Diante disso, outros achados clínicos sugerem que usuários crônicos de substância de abuso, diagnosticados com TEPT, possivelmente escolham a substância de abuso conforme seu mecanismo de ação farmacológico ${ }^{24}$, apontando para a hipótese de automedicação como forma de aliviar o so- frimento relacionado ao evento traumático e "apagar" a memória intrusiva e perturbadora ${ }^{2,15,24}$.

A partir de outras investigações a respeito da associação entre trauma, sintomas de TEPT e sintomas de dependência, estudos demonstram que uma melhora/piora na intensidade e frequência de uso de cocaína se associa com a melhora/piora dos sintomas de TEPT 25,26. Por exemplo, o estudo de Wasserman et al. com dependentes de cocaína indicou que $69 \%$ das pacientes relataram que a piora nos sintomas póstraumáticos ocasionava igualmente uma piora nos sintomas associados à dependência de cocaína ${ }^{26}$. Ainda, considerando os pressupostos de conhecimento referente a trauma e dependência, alguns autores sugeriram que o TEPT possivelmente seja um fator de risco para a adição a drogas de abuso, embora nenhum dos estudos associativos evidencie que a dependência possa ser considerada fator de risco ao diagnóstico pós-traumático ${ }^{15,27}$.

Mesmo que não se possa afirmar que não ocorra uso ou abuso de substâncias antecedentes ao desenvolvimento do TEPT28,29, alguns estudos buscaram pesquisar o impacto da experiência traumática na busca e consumo de drogas de abuso, investigando possíveis diferenças quanto à idade de ocorrência do trauma e às implicações posteriores ${ }^{27,30}$. Nesse sentido, constataram que um número significativo de sujeitos com dependência de substâncias vivenciou algum evento traumático nos primeiros anos de vida e que esses sujeitos apresentaram alterações referentes à idade do primeiro uso de substâncias, experimentando diversas drogas prematuramente em relação a sujeitos que vivenciaram uma experiência traumática somente na idade adulta ${ }^{11,31}$. Segundo esses estudos, quanto mais precoce o trauma vivenciado, mais prematuro poderia ser o primeiro contato com as substâncias de abuso consideradas "porta de entrada" como álcool e maconha, ainda que esse efeito possa estar associado igualmente à experimentação de cocaína tipo $\mathrm{crack}^{30}$.

A importância de estudos relacionando a dependência de substâncias psicoativas em pacientes com TEPT está fundamentada na literatura. Entretanto, existe uma lacuna no que se refere a estudos com usuários de cocaína tipo crack. Diante disso, o objetivo do presente estudo foi verificar a ocorrência de TEPT em uma amostra de mulheres dependentes de cocaína tipo crack, assim como a presença dos distintos clusters de sintomas pós-traumáticos, além de descrever os tipos de eventos vivenciados pelas usuárias. Além disso, buscou-se investigar se os eventos traumáticos que ocorrem precocemente influenciam na idade do primeiro uso de substâncias.

\section{MÉTODO}

\section{Amostra}

A amostra foi composta por 99 mulheres usuárias de crack, acompanhadas durante 20 dias de tratamento para 
desintoxicação por uso de crack em uma unidade pública de internação psiquiátrica especializada. A participação foi voluntária e anônima, a partir da assinatura de um Termo de Consentimento Livre e Esclarecido (TCLE) previamente aprovado pelo Comitê de Ética e Pesquisa das instituições participantes. Foram elegíveis para o estudo mulheres entre 18 e 55 anos com diagnóstico de dependência por cocaína tipo crack (segundo critérios DSM-IV-TR), com escolaridade mínima de quatro anos, ausência de síndromes psicóticas comórbidas, além do escore acima de 18 no Miniexame do Estado Mental, conforme sugestão para pessoas de baixa escolaridade ${ }^{32,33}$. Os critérios de exclusão foram: presença de sintomas psicóticos previamente não detectados, doenças neurológicas, infecciosas e metabólicas conhecidas, déficits cognitivos graves que implicassem em alteração do estado de consciência e agitação psicomotora.

Para investigar o efeito da idade do primeiro evento traumático na idade do primeiro uso de drogas de abuso, dividiu-se a amostra em dois grupos: Grupo Trauma Precoce (TP) e Grupo Trauma na Adultez (TA). Para isso, as participantes que vivenciaram algum evento de natureza traumática antes dos 18 anos foram incluídas no grupo TP e as demais, expostas a trauma apenas na vida adulta, foram incluídas no grupo TA.

\section{Instrumentos}

Para investigação de diagnósticos psiquiátricos, foi utilizada a SCID-I (Structured Clinical Interview for DSM-IM) ${ }^{34}$. Esse instrumento, fundamentado nos critérios diagnósticos do DSM-IV-TR, contempla a investigação de sintomas correspondentes aos transtornos de eixo I. Como o presente estudo se propôs a delinear cuidadosamente a exposição a situações potencialmente traumáticas, além dos critérios e sintomas correspondentes ao TEPT, todas as questões foram abordadas, independentemente de a paciente indicar a presença ou ausência do sintoma e/ou critério, mesmo que o instrumento sugerisse pela não continuidade da aplicação.

O Addiction Severity Index (ASI-6), Escala de Gravidade de Dependência ${ }^{35}$ versão 6 traduzida e adaptada para o Brasili ${ }^{36}$, foi utilizado para obter informações sobre o perfil de uso de substâncias das pacientes, incluindo a história de uso de drogas e o período de início. Trata-se de uma entrevista semiestruturada, com duração de 45 a 60 minutos. Como diferencial, a ASI apresenta uma abordagem multidimensional, representando a estimativa da realidade do entrevistado, contemplando inclusive o histórico de vivências traumáticas. Assim, a sessão referente a trauma foi utilizada como forma de complementar as informações associadas à exposição a eventos estressores e traumáticos. As 15 questões sobre o histórico traumático possibilitaram a categorização quanto à natureza dos eventos traumáticos em cinco agrupamentos distintos: agressão/abuso físico; agressão/abuso sexual; ameaça à vida; ser vítima de crime violento; testemunhar evento traumático.

\section{Procedimentos}

A equipe de pesquisa foi composta por pesquisadores treinados extensivamente para a aplicação dos instrumentos. Após revisão dos prontuários das pacientes internadas, aquelas que atendessem aos critérios de inclusão eram convidadas, sendo informadas conforme o TCLE, configurando uma amostragem por conveniência. A partir do quarto dia de internação, as participantes eram avaliadas quanto a indícios de prejuízo cognitivo grave (MEEM). Após 15 dias de internação, eram aplicadas a SCID-I e a ASI-6.

\section{Análise estatística}

Os dados foram organizados em um banco de dados e analisados por meio do programa SPSS for Windows 17.0. A normalidade das variáveis foi avaliada para todas as variáveis e, quando necessário, o teste T de Student, Mann-Whitney ou qui-quadrado foi utilizado na comparação entre o grupo TP e TA.

\section{RESULTADOS}

\section{Características da amostra}

Entre as 99 participantes incluídas no estudo, 86,9\% ( $n=86)$ reportaram terem sido expostas a algum tipo de evento estressor e potencialmente traumático ao menos uma vez ao longo da vida, caracterizando o critério A1 para TEPT segundo DSM-IV-TR, enquanto 13,1\% relataram a não exposição a eventos dessa natureza. As variáveis sociodemográficas da amostra exposta a eventos traumáticos, bem como do grupo TP e TA, estão expressas na tabela 1.

\section{Prevalência de TEPT e clusters pós-traumáticos}

Das 86 participantes expostas a eventos considerados traumáticos, 15,1\% preencheram todos os critérios para TEPT, por meio da SCID-I. Dessas, 53,8\% $(n=7)$ preencheram critério para TEPT passado e $46,2 \%(n=6)$, para TEPT atual. Verificou-se entre os clusters pós-traumáticos uma presença maior dos sintomas de revivescência e hiperexcitabilidade em comparação com aqueles relacionados à evitação (Tabela 2).

\section{Eventos experienciados}

Quanto à natureza dos eventos vivenciados pelas participantes, agressão/abuso físico $(67,6 \%)$ e ser testemunha de um evento ocorrido a terceiros (57\%) foram os mais frequentemente reportados (Tabela 3).

\section{Relação entre evento traumático e idade do primeiro uso de substâncias}

Com relação à média de idade inicial do uso de substância e a média de idade de exposição ao evento traumático, observou-se que o trauma sucede ao primeiro uso de álcool e maconha e antecede o primeiro uso de cocaína e cocaína tipo crack (Figura 1). 
Tabela 1. Características da amostra

\begin{tabular}{|c|c|c|c|c|c|c|c|c|c|}
\hline & \multicolumn{2}{|c|}{ Total $(n=86)$} & \multicolumn{2}{|c|}{ Trauma precoce $(n=45)$} & \multicolumn{2}{|c|}{ Trauma na adultez $(n=41)$} & \multirow{2}{*}{ Estatística } & \multirow{2}{*}{$g l /(z)$} & \multirow{2}{*}{$p$} \\
\hline & Média/(n) & $\mathrm{DP} /(\%)$ & Média/(n) & $\mathrm{DP} /(\%)$ & Média/(n) & $\mathrm{DP} /(\%)$ & & & \\
\hline \multicolumn{10}{|l|}{ Sociodemográfica } \\
\hline Idade & 29,86 & 7,9 & 28,67 & 7,95 & 31,26 & 7,70 & $t=1,591$ & 84 & .109 \\
\hline Etnia (caucasiano) & $(28)$ & $(32,6)$ & $(12)$ & $(26,7)$ & (16) & (39) & $x^{2}=1,49$ & 1 & .222 \\
\hline Escolaridade & 8,1 & 3,21 & 8,04 & 2,97 & 8,14 & 3,54 & $t=0,176$ & 83 & .890 \\
\hline Renda (R\$) & $1.247,97$ & $1.055,53$ & 1.125 & 872 & 1.383 & 1.223 & $t=1,108$ & 80 & .284 \\
\hline Situação conjugal & & & & & & & $x^{2}=2,48$ & 3 & .478 \\
\hline Solteira & $(50)$ & $(58,1)$ & $(24)$ & $(53,3)$ & $(26)$ & $(63,4)$ & & & \\
\hline Casada/companheira fixa & $(21)$ & $(24,4)$ & (14) & $(31,1)$ & (7) & $(17,1)$ & & & \\
\hline Divorciada & (13) & $(15,1)$ & (6) & $(13,3)$ & (7) & $(17,1)$ & & & \\
\hline Viúva & $(2)$ & $(2,3)$ & (1) & $(2,2)$ & (1) & $(2,4)$ & & & \\
\hline Situação ocupacional & & & & & & & $x^{2}=5,14$ & 2 & .080 \\
\hline Estudante & (4) & $(4,7)$ & (3) & $(6,7)$ & (1) & $(2,4)$ & & & \\
\hline Empregada & $(21)$ & $(23,3)$ & (7) & $(15,6)$ & (14) & $(34,1)$ & & & \\
\hline Desempregada & (61) & $(70,9)$ & $(35)$ & $(77,8)$ & $(26)$ & $(63,4)$ & & & \\
\hline Idade da vivência traumática & 17,81 & 10,77 & 10,33 & 4,16 & 26,76 & 9,26 & $t=9,950$ & 49,21 & .000 \\
\hline \multicolumn{10}{|l|}{ Idade do $1^{\circ}$ uso } \\
\hline Álcool & 15,55 & 5,17 & 14 & 3,89 & 17,14 & 5,85 & $U=530$ & $(-3,41)$ & .001 \\
\hline Maconha & 16,22 & 4,61 & 16,65 & 5,08 & 15,66 & 2,78 & $U=886$ & $(-0,31)$ & .754 \\
\hline Cocaína & 19,69 & 5,94 & 19,92 & 6,41 & 19,03 & 5,44 & $U=921$ & $(-0,01)$ & .990 \\
\hline Crack & 24,21 & 7,52 & 22,44 & 7,03 & 25,09 & 7,45 & $U=610$ & $(-2,70)$ & .007 \\
\hline
\end{tabular}

Tabela 2. Prevalência ao longo da vida de TEPT e dos clusters póstraumáticos

\begin{tabular}{lcc}
\hline Prevalência & $\%$ & $\mathrm{n}=86$ \\
\hline TEPT & 15,1 & 13 \\
Critério B - Revivescência & 24,4 & 21 \\
Critério C - Evitação & 18,6 & 16 \\
Critério D - Hiperexcitabilidade & 20,9 & 18 \\
\hline
\end{tabular}

Tabela 3. Tipo de evento relatado pelas usuárias de cocaína tipo crack

\begin{tabular}{lcc}
\hline Tipos de eventos & $\%$ & $\mathrm{n}=86$ \\
\hline Ameaça a vida ou integridade física & 31,4 & 27 \\
Agressão/abuso físico & 67,4 & 58 \\
Agressão/abuso sexual & $43 \%$ & 37 \\
Testemunhar evento traumático ocorrido com um terceiro & 57 & 49 \\
Ser vítima de crime violento & $32,6 \%$ & 28 \\
\hline
\end{tabular}

Na comparação entre o grupo TP $(n=45)$ versus TA $(n=$ 41), observaram-se diferenças significativas no que se refere à idade de primeiro uso de álcool e de crack (Figura 2).

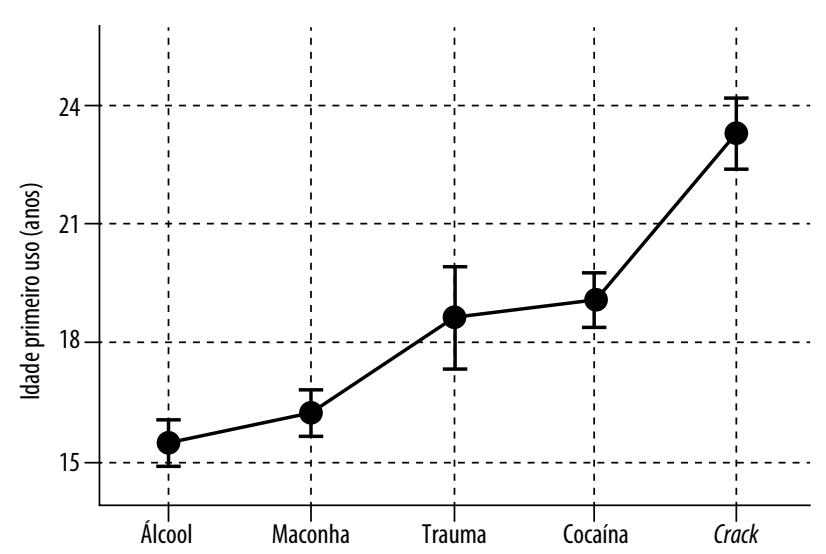

Figura 1. Idade de primeiro uso de substâncias e idade da experiência traumática.

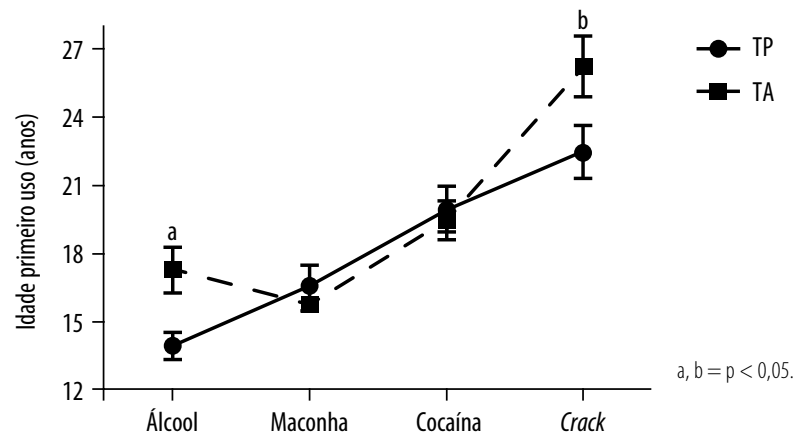

Figura 2. Comparação entre os grupos Trauma Precoce (TP) e Trauma na Adultez (TA) em relação à idade de primeiro uso de substâncias. 


\section{DISCUSSÃO}

O presente estudo buscou investigar a prevalência de TEPT ao longo da vida em uma amostra composta por mulheres dependentes de cocaína tipo crack, em uma unidade pública de internação para desintoxicação, investigando os diferentes sintomas pós-traumáticos, bem como o impacto do trauma precoce no primeiro uso de múltiplas drogas. Nossos achados visam contribuir para o desenvolvimento científico na área da dependência do crack, para que, dessa forma, modelos de intervenções mais eficazes e efetivos possam ser propostos.

De acordo com os resultados, mais de $85 \%$ da amostra atendeu ao critério A1 referente à sintomatologia do TEPT, indicando que a grande maioria das participantes foi exposta a, ao menos, um evento de natureza traumática. Tais dados estão de acordo com estudos anteriores, que indicaram elevadas taxas (superiores a 90\%) de exposição a vivências traumáticas em amostras compostas por usuários ou dependentes de substâncias ${ }^{37,38}$, embora outros estudos tenham demonstrado taxas de exposição inferiores, que variaram entre $36 \%$ e $44 \% 18,39$. Essas diferenças possivelmente decorrem de variações metodológicas entre os estudos citados, por exemplo: a) Nos estudos que apresentaram taxas mais elevadas, as amostras eram compostas por sujeitos com diagnóstico de dependência de substância; b) Nos estudos com taxas inferiores, as amostras eram compostas tanto por dependentes como por usuários que não atendiam a todos os critérios para dependência; c) Amostras compostas por usuários de diferentes substâncias; d) Variações nas características sociodemográficas amostrais. O diferencial do presente estudo foi investigar uma população de mulheres que atendia aos critérios para dependência de cocaína tipo crack, uma vez que esses fatores corroboram um risco maior à exposição a eventos de vida traumáticos.

Por outro lado, estudos com populações gerais demonstraram taxas igualmente elevadas de exposição a eventos potencialmente traumáticos ao longo da vida. Breslau investigou uma amostra representativa da população americana, composta de 34.653 participantes, e encontrou uma prevalência elevada de exposição a eventos traumáticos, assim como o presente estudo ${ }^{40}$. Entretanto, é necessário considerar a natureza dos eventos, visto que na presente amostra o abuso sexual e físico corresponde a 43\% e 67\% respectivamente, o que não ocorre no estudo de Breslau, no qual as taxas referentes aos mesmos eventos foram inferiores, $7 \%$ e $12 \%$. Além disso, sugere-se que a amostra aqui investigada frequentemente vivencie eventos de gravidade e intensidade extrema e possivelmente não reporte como potencialmente traumáticos aqueles eventos que a população em geral avalie como estressor perante o questionamento do critério A1 do DSM-IV-TR. Outra hipótese levantada é a falta de padronização do critério A1 de TEPT, que dificulta a precisão dos tipos de eventos investigados ${ }^{41}$.
No que se refere à prevalência de TEPT, os resultados indicaram que 15,1\% da amostra atendem aos critérios ao longo da vida para o transtorno, e em torno de 50\% mantêm a sintomatologia pós-traumática acentuada. Estudos prévios apresentaram taxas de prevalência de TEPT variando entre $10 \%$ e 50\% $\%^{16,18,19,38,39}$, o que indica que os resultados desse estudo estão de acordo com os índices apresentados. Contudo, a taxa de prevalência encontrada é inferior à média dos demais, possivelmente pelas variações metodológicas já elucidadas. Outra provável explicação se deve à utilização da SCID-I para investigação diagnóstica, instrumento baseado nos critérios nosológicos do DSM-IV-TR, que qualifica um evento potencialmente traumático também como eliciador de uma resposta emocional subjetiva de intenso medo, impotência ou horror (critério A2). Dessa maneira, considerando que a SCID-I é uma entrevista fundamentada no autorrelato do paciente, a memória retrospectiva da resposta emocional associada ao trauma estaria menos suscetível à recordação, uma vez que o evento pode ter ocorrido há muitos anos. Em contrapartida, a maioria dos estudos que apontam elevados índices de prevalência de TEPT utilizou como base para o diagnóstico pós-traumático os critérios do DSM-III-R, que não contempla o critério $\mathrm{A} 2^{39}$.

Quanto aos tipos de eventos potencialmente traumáticos experienciados, os mais relatados pelas pacientes foram de natureza agressão/abuso físico (67\%). O resultado fortalece os estudos anteriores que apontam esse tipo de evento entre um dos mais citados em suas amostras ${ }^{15,26,39}$. Além disso, por causa de os usuários de crack apresentarem comportamentos impulsivos e de exposição a risco, combinado com um ambiente geralmente violento, a probabilidade de sofrer ou testemunhar eventos traumáticos é potencializada. Dessa forma, testemunhar um evento traumático ocorrido com um terceiro apareceu como a segunda natureza de evento mais prevalente (57\%), embora esse achado não tenha sido constatado nos estudos acima citados.

As análises relacionadas aos clusters de sintomas pós-traumáticos indicaram que os clusters B (revivescência) e D (hiperexcitabilidade) foram os mais frequentes, com taxas de 24,4\% e 20,9\%, respectivamente. Esses achados são semeIhantes aos encontrados por Cottler et al. ${ }^{18}$. Uma das hipóteses para a frequência inferior (18,6\%) de sintomas do cluster C (evitação) possivelmente decorre da presença de sintomas de natureza distinta nesse agrupamento. Esse cluster abrange sintomas relacionados à evitação, como evitação de pensamentos, sentimentos, lugares, pessoas ou conversas associadas ao trauma, além de sintomas de entorpecimento, caracterizados pela sensação de afastamento em relação a pessoas, afeto restrito, sentimento de futuro abreviado e redução acentuada de interesse. Percebe-se que esse agrupamento não se restringe apenas aos comportamentos evitativos, mas estende-se a manifestações de humor depressivo. Nesse sentido, tal dicotomia vem sendo uma das temáticas 
discutidas na reformulação do diagnóstico de TEPT para o DSM-V, no qual ocorreria uma separação dos sintomas do critério $C$ em dois diferentes clusters, sendo um específico de comportamentos evitativos e o outro associado a alterações negativas na cognição e humor ${ }^{42}$.

Os dados referentes à idade de início de uso de substâncias demonstraram que o trauma sucede a experimentação de álcool e maconha e antecede a experimentação de cocaína e cocaína tipo crack. Tal achado está de acordo com estudo anterior, em que a exposição traumática antecedeu o primeiro uso de $\mathrm{crack}^{39}$. Entretanto, evidenciou-se diferença no que tange ao primeiro uso de cocaína, em comparação ao estudo de Sack. Uma hipótese para tal resultado poderia ser a diferença socioeconômica das amostras, já que o presente estudo foi realizado em uma unidade para desintoxicação pertencente ao Sistema Único de Saúde. Tal hipótese se baseia em estudos que mostram que a realidade do usuário de substâncias no Brasil, principalmente os de baixa renda, possibilita a compra de cocaína tipo crack por um valor consideravelmente menor que o da cocaína em pó1,43.

Diversos autores também sugerem que as elevadas taxas de comorbidade entre uso de substâncias e TEPT decorram da busca pelo alívio dos sintomas pós-traumáticos por meio dos efeitos psicoativos dessas substâncias ${ }^{44,45}$, assim uma das hipóteses emergentes refere-se à automedicação. Dessa forma, indivíduos com histórico de trauma fariam uso ou abuso de substâncias com objetivo de ajudar a diminuir os sintomas de revivescência (por exemplo, memórias intrusivas e flashbacks) e aliviar as emoções dolorosas (por exemplo, raiva e tristeza) ou sensações físicas (hipervigilância) ${ }^{44}$. Sendo assim, substâncias com propriedades farmacológicas depressoras, como o álcool, podem proporcionar alívio temporário dos estados emocionais desregulados associados ao TEPT. No que tange ao uso da cocaína tipo crack, sabe-se que seus efeitos psicotrópicos, diferente da cocaína inalada, fazem com que o usuário sofra ao extremo com seus sentimentos e apresente uma dificuldade em controlar ou experimentar suas emoções, por vezes até mesmo ausentando-se ou sentindo-se confuso ${ }^{44,46,47}$, o que poderia corroborar, em curto prazo, a hipótese de automedicação. Entretanto, o uso crônico da cocaína tipo crack teria como consequência a intensificação dos sintomas de entorpecimento emocional e humor deprimido ${ }^{48}$.

No que se refere à idade em que os indivíduos vivenciaram o evento traumático, estudos prévios demonstraram que experiências adversas nos primeiros anos de vida parecem ter uma forte associação com o desenvolvimento de comportamentos impulsivos e de ordem motivacional perseverantes, principalmente aqueles relacionados à busca por estímulos prazerosos ${ }^{49}$. Dentre essas experiências adversas, estudos associam, principalmente, o abuso sexual com o início precoce do uso de substância ${ }^{50,51}$. Uma das consequências dessas alterações é o aumento na probabilidade de uso de substâncias psicoativas, assim como a antecipação do período de contato com essas substâncias. Nossos achados estão de acordo com o exposto, uma vez que as participantes incluídas no grupo TP apresentaram médias de idade de primeiro uso significativamente inferiores no que se refere ao álcool e à cocaína tipo crack em comparação ao grupo TA. Nesse sentido, a experiência traumática na infância pode ser considerada um fator de risco para a experimentação de drogas e subsequentemente ao desenvolvimento da dependência, à medida que a substância poderia ser utilizada como uma tentativa de automedicação para atenuar os sintomas de humor associados ao trauma ${ }^{52}$. Embora não se tenha observado tal associação na idade de início de uso de cocaína e maconha, sugere-se que o impacto do trauma na infância contribua diretamente para a experimentação de álcool, uma vez que estudos apontam para ele como a porta de entrada para o uso e abuso de substâncias e, consequentemente, uma busca por uma droga potente e economicamente mais viável, como o crack, mais precocemente ${ }^{4,30}$. Além disso, o próprio abuso de substâncias em idade precoce tornaria o indivíduo mais vulnerável a uma resposta exacerbada associada a uma vivência potencialmente traumática ${ }^{27}$.

Nesse momento, cabe ressaltar que o estudo possui algumas limitações: a) Conforme viés de Berkson ${ }^{53}$ é importante considerar inter-relação entre TEPT e TRUS na amostra estudada, uma vez que amostras de pacientes internados em hospitais podem ser tendenciosas quando se realizam estudos de comorbidade. Em virtude disso, os presentes resultados devem ser considerados de forma cautelosa no que diz respeito à generalização para toda população de usuários de drogas ilícitas ${ }^{53}$; b) Não foram utilizados questionários com a finalidade de avaliar a intensidade e a gravidade dos sintomas pós-traumáticos, embora se recomende que estudos futuros possam avaliar também esses fatores; c) O número de eventos traumáticos vivenciados pelas participantes não foi investigado, embora se considere a possibilidade de que as participantes que foram expostas a eventos traumáticos precocemente sejam também aquelas que foram expostas ao maior número de eventos; d) Apesar de a ASI-6 ser uma entrevista semiestruturada e ter como objetivo organizar diversas informações referentes ao perfil do usuário de substâncias, considerou-se a presença de limitações associadas a essa entrevista, uma vez que alguns dados, como a idade de início de uso das substâncias, necessitam que o entrevistado pontue objetivamente períodos de tempo específicos. Desse modo, essas informações estavam suscetíveis a vieses de memória e à falta de fidedignidade, sobretudo considerando os já conhecidos déficits de memória retrospectiva associados tanto a dependência de substâncias como ao TEPT ${ }^{54}$.

Por fim, considerando que o correto diagnóstico e a investigação das comorbidades nos transtornos psiquiátricos são de fundamental importância para bons resultados nos 
tratamentos, estudos indicam um quadro clínico mais grave em usuários de substâncias com histórico de traumas ${ }^{11,39}$. Nesse sentido, os pacientes diagnosticados com TEPT podem apresentar características que impeçam a evolução do tratamento focado na dependência do crack, uma vez que, antes mesmo de iniciar o manejo comportamental associado ao uso da droga, esses indivíduos já possuem prejuízos procedentes da sintomatologia pós-traumática. A intervenção eficaz e direta deve ser capaz de propor o esbatimento desses sintomas. Isso poderia favorecer os processos de mudança e redução dos comportamentos disfuncionais da dependência de cocaína tipo crack. Dessa forma, o reconhecimento precoce dessa comorbidade pode prevenir sua cronicidade, favorecer a aderência e promover o tratamento adequado para ambos os transtornos. No caso de ausência da comorbidade pós-traumática, sugere-se, ainda, que seja investigada a história de experiências traumáticas vivenciadas pelos usuários, sobretudo aquelas que ocorreram na infância e adolescência, haja vista a possível associação com o início de uso de drogas e consequências clínicas.

\section{AGRADECIMENTOS}

Agradecemos à Unidade de São Rafael, Sistema de Saúde Mãe de Deus, e a toda sua equipe de profissionais pelo acolhimento e apoio, possibilitando a realização do presente estudo. Além disso, agradecemos também à Coordenação de Aperfeiçoamento de Pessoal de Nível Superior (Capes), ao ConseIho Nacional de Desenvolvimento Científico e Tecnológico (CNPq) e à Fundação de Amparo à Pesquisa do Estado do Rio Grande do Sul (Fapergs) pelo incentivo e fomento à pesquisa.

\section{REFERÊNCIAS}

1. Duailibi LB, Ribeiro M, Laranjeira R. Profile of cocaine and crack users in Brazil. Cad Saude Publica. 2008;24(Supl 4):545-57.

2. Stewart SH, Pihl RO, Conrod PJ, Dongier M. Functional associations among trauma, PTSD, and substance-related disorders. Addict Behav. 1998;23(Supl 6):797-812.

3. Le Moal M. Drug abuse: vulnerability and transition to addiction. Pharmacopsychiatry. 2009;42(Supl 1):42-55.

4. Sacks JY, McKendrick K, Banks $S$. The impact of early trauma and abuse on residential substance abuse treatment outcomes for women. J Subst Abuse Treat. 2008;34(Supl 1):90-100.

5. Easton CJ, Swan S, Sinha R. Prevalence of family violence in clients entering substance abuse treatment. J Subst Abuse Treat. 2000;18(Supl 1):23-8.

6. Jacobsen LK, Southwick SM, Kosten TR. Substance use disorders in patients with posttraumatic stress disorder: a review of the literature. Am J Psychiatry. 2001;158(Supl 8):1184-90.

7. Kessler RC, Sonnega A, Bromet E, Hughes M, Nelson CB. Posttraumatic stress disorder in the National Comorbidity Survey. Arch Gen Psychiatry. 1995;52(Supl 12):1048-60.

8. Breslau N, Davis GC, Andreski P, Peterson E. Traumatic events and posttraumatic stress disorder in an urban population of young adults. Arch Gen Psychiatry. 1991;48(Supl 3):216-22.

9. Breslau N, Davis GC, Peterson EL, Schultz L. Psychiatric sequelae of posttraumatic stress disorder in women. Arch Gen Psychiatry. 1997;54(Supl 1):81-7.
10. Ford JD, Hawke J, Alessi S, Ledgerwood D, Petry N. Psychological trauma and PTSD symptoms as predictors of substance dependence treatment outcomes. Behav Res Ther. 2007:45(Supl 10):2417-31.

11. Farrugia PL, Mills KL, Barrett E, Back SE, Teesson M, Baker A, et al. Childhood trauma among individuals with co-morbid substance use and post traumatic stress disorder. Ment Health Subst Use. 2011;4(Supl 4):314-26.

12. Boudewyns PA, Albrecht JW, Talbert FS, Hyer LA. Comorbidity and treatment outcome of inpatients with chronic combat-related PTSD. Hosp Community Psychiatry. 1991;42(Supl 8):847-9.

13. McFall ME, Mackay PW, Donovan DM. Combat-related PTSD and psychosocial adjustment problems among substance abusing veterans. J Nerv Ment Dis. 1991;179(Supl 1):33-8.

14. Fullilove MT, Fullilove R, Smith M, Winkler K, Michael C, Panzer P, et al. Violence, trauma, and post-traumatic stress disorder among women drug users. J Traum Stress. 2006;6(Supl 4):533-43.

15. Tull MT, Gratz KL, Aklin WM, Lejuez CW. A preliminary examination of the relationships between posttraumatic stress symptoms and crack/cocaine, heroin, and alcohol dependence. J Anxiety Disord. 2010;24(Supl 1):55-62.

16. Brown PJ, Recupero PR, Stout R. PTSD substance abuse comorbidity and treatment utilization. Addict Behav. 1995;20(Supl 2):251-4.

17. Najavits LM, Gastfriend DR, Barber JP, ReifS, Muenz LR, Blaine J, et al. Cocaine dependence with and without PTSD among subjects in the National Institute on Drug Abuse Collaborative Cocaine Treatment Study. Am J Psychiatry. 1998;155(Supl 2):214-9.

18. Cottler LB, Nishith $P$, Compton WM. Gender differences in risk factors for trauma exposure and post-traumatic stress disorder among inner-city drug abusers in and out of treatment. Compr Psychiatry. 2001;42(Supl 2):111-7.

19. Najavits LM, Runkel R, Neuner C, Frank AF, Thase ME, Crits-Christoph P, et al. Rates and symptoms of PTSD among cocaine-dependent patients. I Stud Alcohol. 2003;64(Supl 5):601-6.

20. Dansky BS, Brady KT, Saladin ME. Untreated symptoms of PTSD among cocaine-dependent individuals. Changes over time. J Subst Abuse Treat. 1998;15(Supl 6):499-504.

21. Kosten TR, Krystal J. Biological mechanisms in posttraumatic stress disorder. Relevance for substance abuse. Recent Dev Alcohol. 1988;6:49-68.

22. Satel SL, Price LH, Palumbo JM, McDougle CJ, Krystal JH, Gawin F, et al. Clinical phenomenology and neurobiology of cocaine abstinence: a prospective inpatient study. Am J Psychiatry. 1991;148(Supl 12):1712-6.

23. Weddington WW, Brown BS, Haertzen CA, Cone EJ, Dax EM, Herning RI, et al. Changes in mood, craving, and sleep during short-term abstinence reported by male cocaine addicts. A controlled, residential study. Arch Gen Psychiatry. 1990;47(Supl 9):861-8.

24. Brown PJ, Wolfe J. Substance abuse and post-traumatic stress disorder comorbidity. Drug Alcohol Depend. 1994;35(Supl 1):51-9.

25. Wiechelt SA, Miller BA, Smyth NJ, Maguin E. Associations between Post-Traumatic Stress Disorder Symptoms and Alcohol and Other Drug Problems: Implications for Social Work Practice. Practice (Birm). 2011;23(Supl 4):183-99.

26. Wasserman DA, Havassy BE, Boles SM. Traumatic events and post-traumatic stress disorder in cocaine users entering private treatment. Drug Alcohol Depend. 1997;46(Supl 1-2):1-8.

27. Andersen SL, Teicher MH. Desperately driven and no brakes: developmental stress exposure and subsequent risk for substance abuse. Neurosci Biobehav Rev. 2009;33(Supl 4):516-24.

28. Chilcoat HD, Breslau N. Investigations of causal pathways between PTSD and drug use disorders. Addict Behav. 1998;23(Supl 6):827-40.

29. Breslau N, Davis GC, Schultz LR. Posttraumatic stress disorder and the incidence of nicotine, alcohol, and other drug disorders in persons who have experienced trauma. Arch Gen Psychiatry. 2003 60(Supl 3):289-94.

30. Hyman SM, Garcia M, Sinha R. Gender specific associations between types of childhood maltreatment and the onset, escalation and severity of substance use in cocaine dependent adults. Am J Drug Alcohol Abuse. 2006;32(Supl 4):655-64.

31. Khoury L, Tang YL, Bradley B, Cubells JF, Ressler KJ. Substance use, childhood traumatic experience, and Posttraumatic Stress Disorder in an urban civilian population. Depress Anxiety. 2010;27(Supl 12):1077-86.

32. Bertolucci PH, Brucki SM, Campacci SR, Juliano Y. 0 Miniexame do Estado Mental em uma populaçäo geral: impacto da escolaridade. Arq Neuropsiquiatr. 1994;52(Supl 1):1-7. 
33. Maia AL, Godinho C, Ferreira ED, Almeida V, Schuh A, Kaye J, et al. Aplicação da versão brasileira da Escala de Avaliação Clínica da Demência (Clinical Dementia Rating - CDR) em amostras de pacientes com demência. Arq Neuropsiquiatr. 2006;64(Supl 2B):485-9.

34. Del-Ben CM, Vilela JA, Crippa JA, Hallak JE, Labati CM, Zuardi AW. Reliability of the Structered Clinical Interview for DSM-IV - Clinical Version. J Bras Psiquiatr. 2001;23(Supl 3):156-9.

35. McLellan AT, Luborsky L, Woody GE, O'Brien CP. An improved diagnostic evaluation instrument for substance abuse patients. The Addiction Severity Index. J Nerv Ment Dis. 1980;168(Supl 1):26-33.

36. Kessler FHP, Cacciola J, Faller S, Souza-Formigoni ML, Cruz M, Brasiliano S. Adaptação transcultural multicêntrica da sexta versão da Escola de Gravidade de Dependência (ASI6) para o Brasil. Rev Psiquiatr Rio Gd Sul. 2007;29(Supl 3):335-6.

37. Ford JD, Hawke J, Alessi S, Ledgerwood D, Petry N. Psychological trauma and PTSD symptoms as predictors of substance dependence treatment outcomes. Behav Res Ther. 2007:45(Supl 10):2417-31.

38. Brown PJ, Stout RL, Gannon-Rowley J. Substance use disorder-PTSD comorbidity. Patients' perceptions of symptom interplay and treatment issues. J Subst Abuse Treat. 1998;15(Supl 5):445-8.

39. Johnson SD, Striley C, Cottler LB. The association of substance use disorders with trauma exposure and PTSD among African American drug users. Addict Behav. 2006;31(Supl 11):2063-73.

40. Breslau N, Kessler RC, Chilcoat HD, Schultz LR, Davis GC, Andreski P. Trauma and posttraumatic stress disorder in the community - The 1996 Detroit Area Survey of Trauma. Arch Gen Psychiatry. 1998;55(7):626-32.

41. Luz MP, Mendlowicz M, Marques-Portella C, Gleiser S, Berger W, Neylan TC, et al. PTSD criterion A1 events: a literature-based categorization. J Trauma Stress. 2011;24(3):243-51.

42. Friedman MJ, Resick PA, Bryant RA, Brewin CR. Considering PTSD for DSM-5. Depress anxiety. 2011;28(Supl 9):750-69.
43. Ferreira Filho OF, Turchi MD, Laranjeira R, Castelo A. [Epidemiological profile of cocaine users on treatment in psychiatrics hospitals, Brazil]. Rev Saude Publica. 2003;37(6):751-9.

44. Khantzian EJ. The self-medication hypothesis of addictive disorders: focus on heroin and cocaine dependence. Am J Psychiatry. 1985;142(Supl 11):1259-64.

45. Ouimette P, Read JP, Wade M, Tirone V. Modeling associations between posttraumatic stress symptoms and substance use. Addict Behav. 2010;35(Supl 1):64-7.

46. Hatsukami DK, Fischman MW. Crack cocaine and cocaine hydrochloride. Are the differences myth or reality? JAMA. 1996;276(Supl 19):1580-8.

47. Khantzian EJ. The self-medication hypothesis of substance use disorders: a reconsideration and recent applications. Harv Rev Psychiatry. 1997:4(Supl 5):231-44.

48. Schonnesson LN, Williams M, Atkinson J, Timpson S. Factors associated with depressive symptoms in African American crack cocaine smokers. J Subst Use. 2009;14(Supl 3-4):161-74.

49. Ernst M, Romeo RD, Andersen SL. Neurobiology of the development of motivated behaviors in adolescence: a window into a neural systems model. Pharmacol Biochem Behav. 2009;93(Supl 3):199-211.

50. Kingston S, Raghavan C. The relationship of sexual abuse, early initiation of substance use, and adolescent trauma to PTSD. J Trauma Stress. 2009;22(Supl 1):65-8.

51. Smith DW, Letourneau EJ, Saunders BE, Kilpatrick DG, Resnick HS, Best CL. Delay in disclosure of childhood rape: results from a national survey. Child Abuse Negl. 2000;24(Supl 2):273-87.

52. Langeland W, Draijer N, Van den Brink W. Trauma and dissociation in treatment-seeking alcoholics: towards a resolution of inconsistent findings. Compr Psychiatry. 2002;43(Supl 3):195-203.

53. Coutinho M. Princípios de epidemiologia clínica aplicada a cardiologia. Arq Bras Cardiol. 1998;71(2):109-16

54. Paxton JL, Vassileva J, Gonzalez R, Maki PM, Martin EM. Neurocognitive performance in drug-dependent males and females with posttraumatic stress disorder symptoms. J Clin Exp Neuropsychol. 2012;34(Supl 5):521-30. 